\title{
Integration of Fuzzy Classifiers with Decision Trees
}

\author{
I-Jen Chiang Jane Yung-jen Hsu \\ chiang@robot.csie.ntu.edu.tw yjhsu@csie.ntu.edu.tw \\ Department of Comuter Science and Information Engineering \\ National Taiwan University \\ Taipei, Taiwan 107, R.O.C.
}

\begin{abstract}
It is often difficult to make accurate predictions given uncertain and noisy data for classification. Unfortunately, most real-world problems have to deal with such imperfect data. This paper presents a new model for fuzzy classification by integrating fuzzy classifiers with decision trees. In this approach, a fuzzy classification tree is constructed from the training data set. Instead of defining a specific class for a given instance, the proposed fuzzy classification scheme computes its degree of possibility for each class. The performance of the system is evaluated by empirically compared with a standard decision tree classifier $\mathrm{C} 4.5$ on several benchmark data sets the UCI machine learning repository.
\end{abstract}

\section{Introduction}

Classification techniques such as decision trees ${ }^{4}$ have been widely used for discovering regularities in complex data. Successful applications include process control, pattern recognition, and diagnosis. Most existing classification techniques have difficulty in dealing with uncertain and noisy data ${ }^{5,6,9}$. Unfortunately, for many real world problems, uncertainty and noise in data cannot be ignored.

Previously, we have introduced the concept of fuzzy classification trees $(\mathrm{FCT})^{2}$ for domains with vague classifications. Rather than performing a two-stage process that couples decision trees with either pre-fuzzification 8,9 or post-fuzzification $1,3,7$, the fuzzy classification tree presents a theoretically sound integration of fuzzy classifiers with decision trees. The structure is very robust with respect to a large amount of noise in the data for classification.

In this paper, we will present the algorithm for constructing fuzzy classification trees, as well as some empirical results on five different data sets from the UCI repository. Section 2 briefly reviews the definitions of fuzzy classification trees. Section 3 describes the basic algorithm for constructing a fuzzy classification tree from a data set. The empirical results comparing FCT with $\mathrm{C} 4.5^{4}$ are summarized in section 4 , followed by the conclusion. 


\section{Definitions}

This section introduces the problem of fuzzy classification.

For any classification problem, the collection of all possible instances constitute the instance space, which is denoted by $\mathcal{X}$. Let $\mathcal{C}=\left\{C_{1}, C_{2}, \ldots C_{n}\right\}$ is a set of classes. A classifier or decision function, $D$, is a function that maps each instance into a class. That is, $D(\mathbf{x})=C_{i}$, where $\mathbf{x} \in \mathcal{X}$ and $C_{i} \in \mathcal{C}$

A fuzzy classifier is a function, $\mathbf{F}: \mathcal{X} \longrightarrow\left\{\left\langle\wp_{1}, \ldots, \wp_{n}\right\rangle \mid \wp_{i} \in[0,1]\right\}$, such that each $\wp_{i}$ is a function defining the possibility that an instance belongs to the class $C_{i}$.

A fuzzy classification tree (FCT) is used to implement the fuzzy classifier. Given an FCT, let $N_{L}$ denote the node labelled by $L$, and $B_{L}$ denote the branch leading into node $N_{L}$. The children of $N_{L}$ are labelled as $N_{L . i}$, where $i \in\{1,2,3, \ldots\}$. Each node $N_{L}$ is associated with a class $C_{L}$ and the possibility function $P_{L}$. Each branch $B_{L}$ is associated with a membership function $\mu_{L}$ which is a function that defines the degree of possibility for any instance $\mathbf{x} \in \mathcal{X}$ to be classified as class $C_{L}$ based on attribute $A$.. The possibility function $P_{L}$ is the composition of the membership functions along the branches from root to node $N_{L}$. If $N_{L}$ is the root node, $P_{L}$ is set to be 1 .

In general, there are many FCTs that implement the same fuzzy classifier. The entropy has been used to evaluate the $\mathrm{FCTs}^{2}$. The entropy function of node $N_{L}$ is

$$
\operatorname{Info}\left(S_{L}\right)=-\sum_{\forall c \in \mathcal{C}} \frac{\mathcal{P}_{L}^{c}}{\mathcal{P}_{L}} \times \ln \frac{\mathcal{P}_{L}^{c}}{\mathcal{P}_{L}} .
$$

The entropy, i.e., information content, of $\mathcal{T}_{L}$ can be defined as

$$
\operatorname{Info}_{T}\left(S_{L}\right)=\sum_{i=1}^{b_{L}} \frac{\mathcal{P}_{L, i}}{\mathcal{P}_{L}} \times \operatorname{Info}\left(S_{L, i}\right) .
$$

where $b_{L}$ is number of branches from node $N_{L}, \mathcal{P}_{L}$ be the sum of possibility $P_{L}(\mathbf{x})$ for all $\mathbf{x}$ in node $N_{L}, \mathcal{P}_{L}^{c}$ be the sum of possibility $P_{L}(\mathbf{x})$ for all $\mathbf{x}$ in node $N_{L}$ of class $c \in \mathcal{C}$. The information gain at node $N_{L}$ is defined by

$$
\operatorname{Gain}\left(\text { Test }_{L}\right)=\operatorname{Info}\left(S_{L}\right)-\operatorname{Info}_{T}\left(S_{L}\right)
$$

due to the test Test $_{L}$.

\section{Construction of Fuzzy Classification Trees}

In this section, an algorithm for fuzzy classification is presented. The main algorithm for constructing an FCT is shown in Figure 1. 
Initially, the system is given a set of training instances, $S_{1}$ denoted by $S_{0}$ in it, which is $S_{0}$. Let $\mathcal{L}$ contain the set of labels corresponding to the unexpanded leaf nodes of the tree. Let $S_{L}$ denote the set of instances that have bee assigned to node $N_{L}$. The algorithm starts by creating a root node $N_{1}$, adding its label to $\mathcal{L}$, and initializing $S_{1}$ to be $S_{0}$.

\section{Algorithm BUILD_FCT \\ [Input] A set of real-valued training instances $S_{0}$.}

\section{[Output] An FCT.}

$$
\begin{aligned}
& \text { 1. } L \longleftarrow 1 \\
& \text { 2. } \mathcal{L} \longleftarrow\{1\} \\
& \text { 3. } S_{1} \longleftarrow S_{0} \\
& \text { 4. Until } \mathcal{L}=\phi \\
& \text { 5. } \quad L \longleftarrow \text { random }(\mathcal{L}) \\
& \text { 6. } \quad \mathcal{L} \longleftarrow \mathcal{L} \backslash\left\{N_{i}\right\} \\
& \text { 7. } \quad \forall a_{i}, T_{L} \longleftarrow \text { spawn_new_tree }\left(N_{L}, a_{i}\right) \\
& \text { 8. } \quad \text { Best } \longleftarrow T_{k} \text { s.t. Info( }\left(T_{k}\right)=\max _{j} \operatorname{Info}\left(T_{j}\right) \\
& \text { 9. } \quad \text { Gain } \longleftarrow \text { Info( }\left(T_{L}\right)-\operatorname{Info}(\operatorname{Best}) \\
& \text { 10. } \quad \text { if } \text { Gain }>\epsilon \\
& \text { 11. } \quad \text { Add the labels of all leaf nodes of Best into } \mathcal{L} \\
& \text { 12. } \quad \text { Assign subsets of } S_{L} \text { into } S_{L .1}, \ldots, S_{L . k}
\end{aligned}
$$

Figure 1: The algorithm for constructing an FCT.

The procedure spawn_new_tree $\left(N_{L}, a_{i}\right)$ defines an expansion from node $N_{L}$ according to attribute $a_{i}$. The details of procedure is shown in Figure 2. All attributes of an instance are viewed as coordinates in an $n$-dimensional Euclidean space.

Consider any cluster of class $C_{i}$ along the coordinate $a_{i}$ resulted from step 3. We first calculate its center of gravity by standard geometric method.

Suppose that there are $k$ branches generated by an attribute. The procedure for computing the entropy of any given FCT is defined by the algorithm in Figure 3. 
Algorithm $S P A W N \_N E W_{-} T R E E$

[Input] A leaf node $N_{L}$ and an attribute $a_{i}$.

[Output] A subset expanded from $N_{L}$.

1. $\forall j$ Project instances in $S_{L}$ of class $C_{j}$ onto attribute $a_{i}$

2. Smooth the resulting histograms using $k$-median method

3. Partition each smoothed histogram into clusters

4. Create a new branch from $N_{L}$ for each cluster.

5. Define the membership function for each branch

Figure 2: The spawn_new_tree algorithm.

Algorithm EVALUATE_ENTROPY

[Input] An FCT with root node $N_{L}$.

[Output] The entropy value of $\mathcal{T}_{L}$.

1. $i \longrightarrow 0$

2. $\forall C_{j}$

3. $\quad i \longleftarrow i+1$

4. $P_{L}^{C_{j}}=\sum_{\forall \mathbf{x} \in S_{L}} u_{j}(\mathbf{x})$

5. $\mathcal{P}_{L . i}=\sum_{\forall \mathbf{x} \in S_{L}} u_{i}(\mathbf{x})$

6. $P_{L}=-\sum_{C_{j} \text { at } N_{L}} P_{L}^{C_{j}}$

7. $\operatorname{Info}\left(S_{L}\right)=-\sum_{C_{j}}$ at $N_{L} \frac{P_{L}^{C_{j}}}{P_{L}} \ln \frac{P_{L}^{C_{j}}}{P_{L}}$

8. $\operatorname{Info}_{T}\left(S_{L}\right)=-\sum_{l=1}^{k} \frac{\mathcal{P}_{L . l}}{\mathcal{P}_{L}} \times \operatorname{Info}\left(S_{L . i}\right)$

Figure 3: The algorithm for evaluating the entropy of $\mathcal{T}_{L}$. 


\section{Empirical Results}

We have tested our algorithm on five data sets from the UCI repository (ftp:// ftp.ics.uci.edu/machine-learning-databases).

Golf This is sample data set with 14 artificially generated instances. We will use this data set to compare the trees that generate by C4.5 and FCT. There are two classes: "Play" and "Don't Play". Each instance is constructed by four attributes, which are categorical or numerical, associated with their possible values ${ }^{4}$.

Monks' Problem The three Monks' problems are a collection of three binary classification problems over a six-attribute discrete domain. The classes is either 0 or 1 . 6 categorical value attributes, no missing value. There are noisy data in monk1 and monk2.

Ionosphere This data set is a binary classification task. The radar data was collected by a system in Goose Bay, Labrador. This system consists of a phased array of 16 high-frequency antennas with a total transmitted power on the order of 6.4 kilowatts. The targets were free electrons in the ionosphere. "Good" radar returns are those showing evidence of some type of structure in the ionosphere. "Bad" returns are those that do not; their signals pass through the ionosphere. There are 351 instances with all 34 numerical attributes, no missing value.

The result of comparing the accuracy of FCT with C4.5 on these problems is shown in Table 3. Note the tree that we use to compare is without pruning.

Table 3: Average accuracy between C4.5 and FCT over the golf, monks, and ionosphere problems.

\begin{tabular}{|c|c|c|c|c|c|}
\hline & Golf & Monk1 & Monk2 & Monk3 & Ionosphere \\
\hline \hline C4.5 & $100 \%$ & $76.6 \%$ & $65.3 \%$ & $92.6 \%$ & $96.5 \%$ \\
\hline FCT & $100 \%$ & $86.5 \%$ & $73.4 \%$ & $93.2 \%$ & $96.2 \%$ \\
\hline
\end{tabular}

The clustering method determines the performance of FCT. As the result on the ionosphere problem, the accuracy of FCT is lower than the accuracy of C4.5. Since the values of all the attributes are distributed in $[-1,1]$, the size of the clusters has an effect on the accuracy of FCT. To improve the clustering method is one of our further objectives.

\section{Conclusion}

This paper has presented an algorithm that integrates the fuzzy classifiers with decision trees. The algorithm attempts to expand the FCT while minimizing 
its entropy at each step.

We have compared FCT with $\mathrm{C} 4.5$ with the empirical results of five data sets in the above section. From the noise-free data (Golf) to the data with a great amount of noise (Monk2), the accuracy rate of FCT is better than C4.5. C4.5 classifies an instance into exactly one class. The instances with attribute values around class boundaries are forced to be classified into a single class, which may result in wrong predictions, especially in the noisy domains. Instead of making a rigid classification, it is sometimes necessary to identify more than one possible classifications for a given instance.

FCTs allow multiple predictions to be made, each of which is associated with a degree of possibility. In application domains that involve a large amount of data with uncertainty, such as medicine or business, fuzzy classification trees can serve as a useful tool for generating fuzzy rules or discovery knowledge in database.

1. Z. Chi and H. Yan, "ID3-Derived Fuzzy Rules and Optimized Defuzzification for Handwritten Numeral Recognition," IEEE Trans. Fuzzy Systems, Vol.4, No.1, 1996, 24-31.

2. I. -J. Chiang and J. Y. -j Hsu, "Fuzzy Classification Trees," Proc. of Ninth Internat. Symposium on Artificial Intelligence, Cancon, Mexico, 1996.

3. K. J. Cios and L. M. Sztandera, "Continuous ID3 algorithm with Fuzzy Entropy Measures," Proc. of the Internat. Conference on Fuzzy Systems, 1992, 469-476.

4. J. R. Quinlan, C4.5 programs for machine learning, Morgan Kaufmann, San Mateo, CA, 1993.

5. M. Sugeno and G. T. Kang, "Structure Identification of Fuzzy Model," Fuzzy Sets and Systems, Vol.28, 1988, 15-33.

6. T. Takagi and M. Sugeno, "Fuzzy Identification of Systems and its Applications to Modeling and Control," IEEE Trans. System Man Cybernet., Vol.5, 1985, 116-132.

7. T. Tani, M. Sakoda and K. Tanaka, "Fuzzy Modeling by ID3 Algorithm and its Applications to Prediction of Heater Outlet Temperature," Proc. of Second IEEE Internat. Conference on Fuzzy Systems, 1992, 923-930.

8. R. Weber, "Automatic Knowledge Acquisition for Fuzzy Control Application," Proc. of the Internat. Symposium on Fuzzy Systems, 1992, 9-12.

9. Y. Yuan and M. J. Shaw, "Induction of fuzzy decision trees," Fuzzy Sets and Systems, 69, 1995, 125-139. 